Pesq. Vet. Bras. 29(1):65-70, janeiro 2009

\title{
Abordagem sobre o controle do carrapato Rhipicephalus (Boophilus) microplus no sul do Rio Grande do Sul ${ }^{1}$
}

\author{
Tânia Regina B. Santos ${ }^{2^{*}}$, Nara Amélia R. Farias ${ }^{3}$, Nilton A. Cunha Filho ${ }^{3}$, \\ Felipe G. Pappen ${ }^{4}$ e Itabajara S. Vaz Junior ${ }^{5}$
}

\begin{abstract}
Santos T.R.B., Farias N.A.R., Cunha Filho N.A., Pappen F.G. \& Vaz Junior I.S. 2009. [Studies of the management of the tick Rhipicephalus (Boophilus) microplus in southern Rio Grande do Sul, Brazil.] Abordagem sobre o controle do carrapato Rhipicephalus (Boophilus) microplus no sul do Rio Grande do Sul. Pesquisa Veterinária Brasileira 29(1):65-70. Faculdade de Veterinária, Universidade Federal de Pelotas, Campus Universitário s/n, Cx. Postal 354, Pelotas, RS 96010-900, Brazil. Email: tsantos@ufpel.edu.br

In the southern region of Rio Grande do Sul, cattle become infested with Rhipicephalus (B.) microplus mainly between October and April due to the climatic conditions. In addition to knowing its life cycle, knowledge of parasite's epidemiology is essential to establish management strategies. Epidemiological studies on resistance to acaricides in Rio Grande do Sul as well as in the rest of Brazil are scarce. Moreover, the large geographical area and the structural deficiency with respect to the use and access to databases make reliable data difficult to obtain. The present study surveyed the perception by cattle breeders in the southern region of Rio Grande do Sul in regards of identification of $R$. (B.) microplus populations that are difficult to manage using acaricides, as well as the risk factors for the selection of resistant tick populations. Tick management data on beef cattle in 85 properties of seven municipalities were collected. The results revealed that the difficulty in tick management correlated positively with levels of education of the farm owners (up to elementary school, $\mathrm{OR}=3.67$ and $p=0.01$ ) and with the yearly number of acaricide applications (over four, with $\mathrm{OR}=4.05$ and $p=0.006$ ). These results also suggest that properties with more than 100 beef cattle under extensive farming conditions in the southern region of Rio Grande do Sul show characteristics that may contribute to longer acaricide lifetimes as compared to other regions in the Country.
\end{abstract}

INDEX TERMS: Rhipicephalus (Boophilus) microplus, risk factors, resistance, acaricides, South Brazil.

RESUMO.- Na região sul do Rio Grande do Sul a infestação dos bovinos por Rhipicephalus (Boophilus) microplus ocorre, principalmente, entre os meses de outubro e abril, devido às condições climáticas. Além do

\footnotetext{
${ }^{1}$ Recebido em 10 de dezembro de 2007.

Aceito para publicação em 22 de agosto de 2008.

${ }^{2}$ Faculdade de Veterinária, Universidade Federal de Pelotas (UFPel), Campus Universitário s/n, Cx. Postal 354, Pelotas, RS 96010-900, Brasil. *Autor para correspondência: tsantos@ufpel.edu.br

${ }^{3}$ Departamento de Microbiologia e Parasitologia (DEMP), Instituto de Biologia, UFPel, Pelotas, RS.

${ }^{4}$ Mestrando da Faculdade de Veterinária, UFPel, Pelotas, RS.

${ }^{5}$ Centro de Biotecnologia, UFRGS, Cx. Postal 15005, Av. Bento Gonçalves 9500, Prédio 43421, Campus do Vale, Porto Alegre, RS 91501970, Brasil. E-mail: ita@ cbiot.ufrgs.br
}

conhecimento do ciclo biológico desse parasito, também é fundamental conhecer a epidemiologia, para estabelecer estratégias de controle. No Rio Grande do Sul, e também no Brasil, existem poucos estudos epidemiológicos a respeito da resistência aos acaricidas. Além disso, a grande área geográfica e a deficiência estrutural quanto ao uso e acesso a bancos de dados dificultam a obtenção de dados confiáveis. O presente estudo teve como objetivo realizar um inquérito abordando a percepção dos produtores da região sul do Rio Grande do Sul, quanto à identificação de populações de $R$. (B.) microplus difíceis de controlar com acaricidas e os fatores de risco para a seleção de populações de carrapatos resistentes. Para execução do trabalho foram coletados dados sobre o controle do carrapato de bovinos de corte, em 85 propriedades 
de sete municípios, localizados na região sul do Estado. Os resultados revelaram a existência de associação positiva entre a dificuldade de controlar o carrapato com os acaricidas e o grau de instrução do proprietário (até o ensino fundamental com $\mathrm{OR}=3,67$ e $p=0,01)$ e o número de aplicação de carrapaticida por ano (superior a $4 \mathrm{com}$ $\mathrm{OR}=4,05$ e $p=0,006$ ). Esses resultados indicam também que propriedades com mais de 100 bovinos de corte em criação extensiva, na região sul do rio Grande do Sul apresentam características que podem contribuir para uma maior vida útil dos carrapaticidas do que as verificadas em outras regiões do País.

TERMOS DE INDEXAÇÃO: Rhipicephalus (B.) microplus, fatores de risco, resistência, acaricidas, Rio Grande do Sul, Brasil.

\section{INTRODUÇÃO}

A zona geográfica situada próxima ao paralelo $32^{\circ} \mathrm{S}$ é considerada uma área marginal de ocorrência da espécie $R$. (B.) microplus, pois as baixas temperaturas verificadas no período de junho a setembro dificultam a fase de vida livre do parasito (Brum et al. 1985). A infestação do gado é reiniciada a partir de outubro, por larvas da progênie de fêmeas ingurgitadas desprendidas no final do período favorável, que tiveram seus períodos de pré-postura, postura e eclosão prolongados (Gonzales 2003).

A região sul do Rio Grande do Sul encontra-se nessa área marginal e engloba zonas com semelhança de clima e relevo, que possuem várzeas utilizadas para o cultivo do arroz, região de campos e presença de matas nativas. Caracteriza-se pela policultura e criação de bovinos de corte e leite além da criação de ovinos, segundo Fortes (1962) e Gianotti (1994).

No Brasil, o uso dos acaricidas constitui o principal instrumento de controle do carrapato bovino $R$. (B.) microplus (Farias 1999, Vargas et al 2003). Apesar de ser usado amplamente e há muito tempo, o seu emprego é, sistematicamente, feito de maneira incorreta, sem considerar os conhecimentos básicos do ciclo do parasito, o que permitiria um controle estratégico. O controle estratégico aumentaria a eficiência e prolongaria a vida útil dos produtos (Furlong 1993, Rocha et al. 2006).

Além do conhecimento do ciclo biológico desse parasito, também é fundamental conhecer, os fatores de manejo que podem influenciar na vida útil dos produtos carrapaticidas. Os principais fatores desencadeantes na seleção de indivíduos resistentes envolvem falhas na conservação, diluição e aplicação dos produtos, intervalos e método de aplicação que levam ao uso dos produtos em concentrações não letais aos carrapatos (Sutherst \& Comins 1997). Portanto, são fatores importantes e relacionados ao manejo das drogas que devem ser detectados e corrigidos, a fim de proporcionar uma vida útil mais longa para os produtos ainda eficazes no controle desse ectoparasito.

No Rio Grande do Sul, e mesmo no Brasil os dados epidemiológicos são raros, pois a grande área geográfica proporciona uma grande diversidade de parâmetros a serem analisados, tais como biodiversidade, clima, cultura e nível sócio econômico. Além disso, existe uma deficiência estrutural quanto ao uso e acesso a bancos de dados precisos. A soma desses fatores dificulta análises e pesquisas que poderiam nortear estratégias de controle do parasito. Na maioria das vezes, as pesquisas são realizadas com amostras de conveniência, que são importantes para alguns usos, porém muitas vezes este tipo de amostra não permite a descrição de prevalências e fatores de riscos, impossibilitando desta forma, um conhecimento epidemiológico mais completo da região estudada. Como exemplo, são os dados descritos por Farias (1999) no Rio Grande do Sul, Silva et al. (2000) em Goiás, Souza et al. (2003) no Paraná e por Pereira (2006) no Vale do Paraíba, estado de São Paulo.

Desta forma, o presente estudo teve como objetivo realizar um inquérito abordando a percepção dos produtores quanto a identificação de populações de $R$. (B.) microplus difíceis de controlar com acaricidas em suas propriedades e os fatores de risco para a seleção de populações de carrapatos resistentes na região sul do Rio Grande do Sul.

\section{MATERIAL E MÉTODOS}

Para execução do trabalho foram coletados dados, com questionário próprio, sobre o controle do carrapato $R$. (B.) microplus de bovinos de corte, em sete municípios, localizado na região sul do Rio Grande do Sul (Pedro Osório, Cerrito, Morro Redondo, Capão do Leão, Pelotas, Piratini e Canguçu).

Para o cálculo do tamanho da amostra, foram utilizados dados de propriedades com mais de 100 bovinos de corte, fornecidos pelas Inspetorias Veterinárias, dos municípios estudados, com autorização da Secretaria de Agricultura e Abastecimento, Departamento de Produção Animal, Divisão de Fiscalização e Defesa Sanitária Animal.

Os sete municípios estudados possuem um total de 764 propriedades de bovino de corte com mais de 100 bovinos. Pela análise realizada com o programa Epi info 6.0, o tamanho amostral adequado foi de 85 propriedades com uma prevalência estimada de $50 \%$ de dificuldade no controle do carrapato e nível de confiança de $95 \%$.

As coletas de dados sobre o controle do carrapato (com questionários próprios) foram realizadas em 85 propriedades distribuídas proporcionalmente de acordo com o número absoluto de propriedades de cada município. Entre de janeiro de 2005 e maio de 2007 foram aplicados os questionários para os proprietários ou administradores das propriedades que foram selecionadas de forma aleatória sistemática, onde foram abordadas as variáveis apresentadas no Quadro 1.

Os dados foram organizados em formato de planilhas eletrônicas (Microsoft Excel 2003). Cada um dos potenciais fatores de risco do questionário epidemiológico (variáveis de exposição) foi comparado com a variável categórica (observação dos proprietários, de possuírem em suas propriedades, populações de $R$. (B.) microplus difíceis de controlar com o uso de acaricidas) em tabelas de contingência $2 \times 2$ (Qui-quadrado e Teste Exato de Fisher). A partir desse passo foram eliminadas as variáveis que não mostraram importância estatística $(p>0,25)$. 


\section{Quadro 1. Variáveis abordadas considerando a possibilidade de fatores de risco para dificultar o controle do Riphicephalus (Boophilus) microplus (informação dos produtores)}

\begin{tabular}{|c|c|c|c|}
\hline Variável & \multicolumn{3}{|c|}{ Descrição } \\
\hline Grau de instrução do proprietário & $\square$ Até o primeiro $\mathrm{g}$ & grau $\quad \square$ A partir do s & segundo grau \\
\hline Área total da propriedade & $\square \leq 500$ ha & $\square>500$ ha & \\
\hline Área utilizada para agricultura & $\square>30 \%$ & $\square \leq 30 \%$ & \\
\hline Número de bovinos por hectare & $\square<1$ & $\square \leq 1$ & \\
\hline Raça de bovino predominante & $\square$ Européia & $\square$ Cruza Zebu & \\
\hline Compra lotes de bovinos mais que uma vez por ano & $\square$ Sim & $\square$ Não & \\
\hline Controle de entrada dos animais antes de soltar no campo & $\square \operatorname{Sim}$ & $\square$ Não & \\
\hline Assistência veterinária a propriedade & $\square \operatorname{Sim}$ & $\square$ Não & \\
\hline Freqüência da assistência veterinária & $\square$ Se necessária & $\square$ Periódica & \\
\hline Dificuldade no controle do carrapato ${ }^{a}$ & $\square \operatorname{Sim}$ & $\square$ Não & \\
\hline Número de aplicação de carrapaticidas por ano & $\square \leq 4$ & $\square<4$ & \\
\hline Épocas de aplicação & $\square$ Primavera & $\square$ Verão $\quad \square$ Outono & 1o $\square$ Inverno \\
\hline Modo de aplicação & $\square$ Aspersão e pou & ur-on $\square$ Injetável $\quad \square$ & $\square$ Imersão \\
\hline Critério para aplicação & $\square$ Presença de ca & arrapatos visíveis & $\square$ Prevenção de infestação \\
\hline Utiliza endectocida & $\square$ Sim & $\square$ Não & \\
\hline Utiliza alternativa para o controle do carrapato & $\square \mathrm{Sim}$ & $\square$ Não & \\
\hline Faz tratamento para mosca-dos-chifres & $\square \operatorname{Sim}$ & $\square$ Não & \\
\hline
\end{tabular}

a Variável dependente.

Todas as variáveis com $p$ d" 0,25 foram analisadas por um modelo de Regressão Logística (análise multivariada), usando intervalos de confiança de $95 \%$ com o programa Statistix (versão 1.0, software analítico, 1996), que fornece estimativas exatas e relações das probabilidades (associação que quantifica o relacionamento entre as variáveis de exposição e os resultados). Esse modelo permite investigar como as proporções ou as taxas observadas dependem dos possíveis fatores de risco dentro de um delineamento inteiramente casualizado. Os Odds ratio (OR) foram calculados com intervalo de confiança de $95 \%$, também se obtendo, neste modelo, o valor de $p$ para cada variável.

\section{RESULTADOS E DISCUSSÃO}

Ao analisar a freqüência das respostas obtidas dos proprietários ou administradores das propriedades com mais de 100 bovinos de corte, no Quadro 2, observa-se algumas características regionais que podem contribuir ou minimizar a seleção de populações de $R$. (B.) microplus resistente aos acaricidas.

Entre elas é importante ressaltar, o grau de instrução dos proprietários, onde $61 \%$ dos entrevistados possuem escolaridade igual ou superior ao Ensino Médio. Desta forma, possuem potencialmente um maior acesso à informação e maior senso crítico sobre problemas sanitários do rebanho.

Em $79 \%$ das propriedades estudadas, a área destinada à agricultura é inferior a $30 \%$ do total da propriedade, 0 que potencialmente é desfavorável, já que o consórcio pecuária/agricultura promove um excelente controle da fase de vida livre desse parasito, o que já foi descrito e recomendado por Furlong (1993) e Gonzales (2003).

A região caracteriza-se pela a criação extensiva, sendo que em $86 \%$ das propriedades a lotação das pastagens não excede a um bovino por hectare, sendo esse sistema de criação (extensiva) desfavorável ao desenvolvimento do carrapato, pois diminui a chance de as lar- vas localizarem os bovinos para iniciar o parasitismo.

Observa-se que a criação de bovinos cruza zebu (Bos indicus x Bos taurus), ocorre em $72 \%$ das propriedades abordadas, o que já havia sido descrito por Martins et al. (2002), sendo um parâmetro que diminui os índices de infestação, pois, os bovinos de raças zebuínas ou cruzas são menos sensíveis ao parasitismo pelo $R$.(B.) microplus, de acordo com Gonzales (2003).

Em 49\% das propriedades analisadas, há compra de lotes de bovinos mais de uma vez por ano. A comercialização de gado pode contribuir para a disseminação de populações resistentes aos carrapaticidas, pois os lotes comprados podem ser parasitados por R.(B.) microplus, com resistência aos carrapaticidas diferente da existente na população de carrapatos da propriedade. Por outro lado, em $93 \%$ das propriedades é realizado controle de entrada, ou seja, quando os lotes de bovinos chegam à propriedade, são medicados com endectocidas ou carrapaticidas, o que minimiza a disseminação de populações resistentes.

A assistência veterinária, apesar de presente em $71 \%$ das propriedades, é freqüente somente em $29 \%$, pois, na maioria das propriedades essa a assistência é apenas para procedimentos clínicos e/ou cirúrgicos, quando necessários. Portanto, nesses casos não há um trabalho preventivo de estratégias para o controle sanitário dos rebanhos, o que é bastante desfavorável para o desenvolvimento de qualquer ciclo produtivo.

A maioria dos proprietários (62\%) não observa dificuldades de controlar o carrapato com os carrapaticidas utilizados, sendo que $62 \%$ realizam no máximo quatro aplicações de acaricidas ao ano, padrões favoráveis para região, já que quanto mais freqüente o uso de acaricidas (denominado de pressão carrapaticida), maior a seleção de populações resistentes (Nolan 1990, Kemp et al. 1999, Bianchi et al. 2003 e Vivas et al. 2006). 
Quadro 2. Distribuição das freqüências dos fatores que podem influenciar no controle do carrapato Riphicephalus (Boophilus) microplus

\begin{tabular}{|c|c|c|c|}
\hline Variáveis abordadas & Descrição & $\mathrm{N}$ & $\%$ \\
\hline \multirow{2}{*}{ Grau de instrução do proprietário } & Analfabeto até o Ensino Fundamental & 33 & 39 \\
\hline & A partir do Ensino Médio & 52 & 61 \\
\hline \multirow[t]{2}{*}{ Área total da propriedade } & $\leq 500$ ha & 47 & 55 \\
\hline & $>500$ ha & 38 & 45 \\
\hline \multirow[t]{2}{*}{ Área utilizada para agricultura } & $>30 \%$ & 18 & 21 \\
\hline & $\leq 30 \%$ & 67 & 79 \\
\hline \multirow[t]{2}{*}{ Número de bovinos por hectare } & $<1$ & 73 & 86 \\
\hline & $\geq 1$ & 12 & 14 \\
\hline \multirow[t]{2}{*}{ Raça de bovino predominante } & Cruza Zebu & 61 & 72 \\
\hline & Européia & 24 & 28 \\
\hline \multirow[t]{2}{*}{ Compra lotes de bovinos mais que uma vez por ano } & Sim & 42 & 49 \\
\hline & Não & 43 & 51 \\
\hline \multirow[t]{2}{*}{ Faz controle de entrada desses lotes antes de soltar no campo } & Sim & 79 & 93 \\
\hline & Não & 6 & 7 \\
\hline \multirow[t]{2}{*}{ Assistência veterinária a propriedade } & Sim & 60 & 71 \\
\hline & Não & 25 & 29 \\
\hline \multirow[t]{2}{*}{ Freqüência da assistência veterinária } & Periódica & 25 & 29 \\
\hline & Se necessária & 60 & 71 \\
\hline \multirow[t]{2}{*}{ Dificuldade no Controle do Carrapato } & Sim & 53 & 62 \\
\hline & Não & 32 & 38 \\
\hline \multirow[t]{2}{*}{ Número de aplicação de carrapaticidas por ano } & $\leq 4$ & 51 & 62 \\
\hline & $>4$ & 31 & 38 \\
\hline \multirow[t]{3}{*}{ Modo de aplicação } & Imersão & 50 & 60 \\
\hline & Injetável & 16 & 19 \\
\hline & Aspersão e pour-on & 18 & 21 \\
\hline \multirow[t]{2}{*}{ Critério para aplicação } & Prevenção de infestação & 24 & 28 \\
\hline & Presença de carrapatos visíveis & 61 & 72 \\
\hline \multirow[t]{2}{*}{ Utiliza endectocida } & Sim & 85 & 100 \\
\hline & Não & 0 & 0 \\
\hline \multirow[t]{2}{*}{ Utilizam alternativas para o controle do carrapato } & Sim & 19 & 23 \\
\hline & Não & 62 & 77 \\
\hline \multirow[t]{2}{*}{ Faz tratamento para mosca-dos-chifres } & Sim & 39 & 46 \\
\hline & Não & 45 & 54 \\
\hline
\end{tabular}

O modo de aplicação mais utilizado na região é o de imersão, com uso em $60 \%$ das propriedades. Em outras regiões do País, o método de imersão não é utilizado (Rocha et al. 2006). Segundo Bianchi et al. (2003) um dos fatores que está associado com a resistência de populações de $R$. microplus em Nova Caledônia é o modo de aplicação do acaricida, sendo o método de imersão, o que menos induz à seleção de populações resistentes.

Em $72 \%$ das propriedades o critério para a aplicação foi a presença de carrapatos visíveis (fêmeas adultas semiingurgitadas e ingurgitadas), o que não se constitui na melhor estratégia, já que recomenda-se o tratamento preventivo, quando as fases parasitárias presentes são larvas e ninfas, pois a espoliação dos bovinos causada por esses estágios é pequena, em relação ao volume sangüíneo ingerido pelas fêmeas ingurgitadas (Gonzales 2003). Além disso, Martins et al. (2002) ressaltam a importância do tratamento sincronizado de todos os animais de uma propriedade. Por outro lado, se o acaricida for aplicado em concentração não letal (subdosagem), pode permitir que as fêmeas ingurgitadas realizem postura fértil, o que permitirá a seleção de indivíduos resistentes (Sutherst \& Comins 1997).

Os endectocidas são utilizados em $100 \%$ das propriedades, um fator negativo, pois Vivas et al. (2006), verifi- caram que as propriedades que utilizam lactonas macrociclicas (endectocidas) possuem um risco 5,92 vezes maior de selecionar populações de carrapatos resistentes, em relação a propriedades que não utilizam esse princípio ativo. Além disso, $77 \%$ das propriedades não utiliza nenhuma outro método de controle para o $R$. (B.) microplus, dependendo exclusivamente das drogas (Farias 1999, Vargas et al. 2003), e dessa forma, com a dependência dos produtos químicos as aplicações tornam-se mais freqüentes, e por conseqüência ocorre uma maior pressão de seleção para resistência aos acaricidas (Nolan 1990, Kemp et al. 1999, Bianchi et al. 2003, Vivas et al. 2006).

Das propriedades estudadas, $54 \%$ realizam tratamento para mosca-dos-chifres (Haematobia iritans), Vivas et al. (2006) analisaram esse fato através de regressão logística, entretanto, concluíram que não apresentou significância $(p=0,45)$ para a seleção de populações de carrapatos resistentes.

Além das freqüências citadas anteriormente (Quadro 2), verificou-se também que o princípio ativo mais utilizado na região é o amitraz sendo usado em $54 \%$ das propriedades, o que também foi verificado por Farias (1999) e Vargas et al. (2003) em estudos realizados no Rio Grande do Sul. Seguidos dos endectocidas (29\%), as associa- 
Quadro 3. Análise univariada (Qui-quadrado) para identificar possíveis fatores de risco associados com a observação dos proprietários de possuírem populações de Riphicephalus (Boophilus) microplus difíceis de controlar com acaricidas

\begin{tabular}{|c|c|c|}
\hline \multirow[t]{2}{*}{ Variáveis Independentes } & \multicolumn{2}{|c|}{$\begin{array}{l}\text { ariável Dependente, } \\
\text { dificuldade no con- } \\
\text { trole do carrapato } \\
\text { com acaricidas }\end{array}$} \\
\hline & $\chi^{2}$ & $\mathrm{p}$ \\
\hline Grau de instrução do proprietário ${ }^{a}$ & 6,56 & $0,01^{b}$ \\
\hline Área total da propriedade & 1,08 & 0,29 \\
\hline Área utilizada para agricultura ${ }^{a}$ & 4,28 & $0,038^{b}$ \\
\hline Número de bovinos por hectare & 0,95 & 0,26 \\
\hline Raça de bovino predominante & 0,27 & 0,60 \\
\hline Compra lotes de bovinos mais que uma vez por ano & o 0,96 & 0,33 \\
\hline $\begin{array}{l}\text { Faz controle de entrada desses lotes antes de soltar } \\
\text { no campo }\end{array}$ & 3,9 & $0,05^{b}$ \\
\hline Assistência veterinária à propriedade & 0,08 & 0,77 \\
\hline Freqüência da assistência veterinária & 0,04 & 0,84 \\
\hline \multirow{2}{*}{\multicolumn{3}{|c|}{ Épocas de aplicação }} \\
\hline & & \\
\hline Primavera & 1,76 & $0,16^{b}$ \\
\hline Verão & 1,02 & $0,24^{b}$ \\
\hline Outono & 1,44 & $0,16^{\mathrm{b}}$ \\
\hline Inverno & 0,01 & 0,64 \\
\hline Modo de aplicação & 3,70 & $0,15^{\mathrm{b}}$ \\
\hline Critério para aplicação & 0,95 & 0,32 \\
\hline \multicolumn{3}{|l|}{ Utiliza endectocida ${ }^{\mathrm{C}}$} \\
\hline Utilizam alternativas para o controle do carrapato & 0,00 & 0,98 \\
\hline Faz tratamento para mosca-dos-chifres ${ }^{a}$ & 3,02 & $0,08^{b}$ \\
\hline
\end{tabular}

a Fatores de risco com pd"0,05, ou seja, representativo dentro da população estudada.

b Para a analise multivariada foram utilizadas as variáveis com p d" 0,25 .

c Não foi possível o cálculo, pois $100 \%$ dos produtores utilizam endectocida.

Quadro 4. Análise multivariada (Regressão Logística) para identificação de associação dos fatores de risco com as propriedades onde Riphicephalus (Boophilus) microplus é considerado difícil de controlar com acaricidas

\begin{tabular}{lcrr}
\hline Variáveis & Odds Ratio IC (95\%) & $p$ \\
\hline Grau de Instrução & 13,67 & $1,35-9,94$ & 0,01 \\
$0 \geq$ de Ensino Médiob & & & \\
$\quad \begin{array}{llll}\text { analfabeto ou até Ensino Fundamental } \\
\text { Número de aplicação de carrapaticida por ano }\end{array}$ & 14,05 & $1,49-11,03$ & 0,006 \\
& $0 \leq 4^{\mathrm{b}}$ \\
$1>4$ & & & \\
\hline
\end{tabular}

a $I C=$ Intervalo de Confiança de $95 \%$.

b Variável usada como referência.

ções de piretróides sintéticos com organofosforados (14\%), e, baixas freqüências as associação de amitraz com organofosforados, fipronil e fluazuron.

No Quadro 3, pode ser observado, que entre os itens analisados, usando tabelas da contingência $2 \times 2$, há cinco fatores com $p$ d" 0.05: grau de instrução do proprietário, área utilizada para a agricultura, controle de entrada dos lotes de bovinos comprados antes de soltar no campo, número de aplicações de carrapaticida por ano e tratamento para a mosca-dos-chifres (Haematobia irritans).
Esses fatores são representativos dentro da população estudada, conforme já discutido.

As variáveis independentes já citadas e as outras com $p$ d" 0,25 (época de aplicação de acaricida e modo de aplicação) foram utilizadas para a análise multivariada (regressão logística).

O modelo final da regressão logística revelou a existência de associação positiva entre a variável dependente (dificuldade de controlar o carrapato com os acaricidas) com duas variáveis independentes (grau de instrução do proprietário e número de aplicação de carrapaticida por ano), que pode ser observado na Quadro 4.

Os proprietários analfabetos ou com Ensino Fundamental têm 3,67 mais chances de ter dificuldade de controlar o carrapato bovino com os acaricidas, do que os proprietários que possuem grau de instrução superior ao Ensino Médio, com uma diferença estatística de $p=0,01$.

As propriedades que realizam mais de quatro aplicações de acaricidas por ano têm 4,05 vezes mais chances de selecionar populações de carrapatos difíceis de controlar, do que as que aplicam acaricidas no máximo quatro vezes ao ano, sendo o $p=0,006$. Dados similares foram identificados por Vivas et al (2006) segundo os quais, um número de tratamentos igual ou superior a seis ao ano representa um risco maior de selecionar populações de carrapatos resistentes aos acaricidas $(p=0,05)$.

\section{CONCLUSÕES}

No estudo, restrito a propriedades com mais de 100 bovinos de corte, conclui-se que os fatores de risco para a seleção de populações de carrapato difíceis de controlar com acaricidas são: o nível de escolaridade até o ensino fundamental e o número de aplicações de carrapaticidas igual ou maior que quatro ao ano.

Foi também observado que, na população estudada na região sul do Rio Grande do Sul, $61 \%$ dos proprietários possuem escolaridade superior ao ensino médio e $62 \%$ deles realizam um número inferior a quatro aplicações por ano de acaricidas.

Esta analise permite concluir que os métodos de controle utilizados nas propriedades estudadas na região sul do Rio Grande do Sul, possuem características, que podem contribuir para que os acaricidas tenham uma vida útil maior do que em outras regiões do País.

\section{REFERÊNCIAS}

Bianchi M.W., Barré N. \& Messad S. 2003. Factors related to cattle infestation level and resistance to acaricides in Boophilus microplus tick populations in New Caledonia. Vet. Parasitol. 112:75-89.

Brum J.G.W., Gonzales J.C. \& Petruzzi M.A. 1985. Postura e eclosão de Boophilus microplus em diferentes localizações geográficas do RS, Brasil. Arq. Brasil. Med. Vet. Zootec. 37(6):581-587.

Farias N.A.R. 1999. Situación de la resistência de la garrapata Boophilus microplus em la región sur de Rio Grande Del Sur, Brazil. Anais IV Seminário Internacional de Parasitologia Animal, Puerto Vallarta, México, p.25-30.

Fortes A.B. 1962. Compêndio de Geografia Geral do Rio Grande do Sul. $2^{\underline{a}}$ ed. Sulina, Porto Alegre. 
Furlong J. 1993. Controle do carrapato dos bovinos na região Sudeste do Brasil. Bolm Téc. 8, Escola de Veterinária, UFMG, Belo Horizonte, p.40-61.

Gianotti C.A. 1994. A fisionomia do Rio Grande do Sul. $3^{\text {a }}$ ed. Unisinos, Porto Alegre. 473p.

Gonzales J.C. 2003. O Controle do Carrapato do Boi. $3^{\text {a }}$ ed. Universidade de Passo Fundo, RS. 128p.

Kemp D.H., McKenna R.V., Thullner R. \& Willadsen P. 1999. Strategies for tick control in a world of acaricide resistance. Anais IV Seminário Internacional de Parasitologia Animal, Puerto Vallarta, México, p.1-10.

Martins J.R., Evans D.E., Ceresér V.H. \& Correa B.L. 2002. Partial strategic tick contrl within a herdo f European breed cattle in the state of Rio Grande do Sul, southern Brazil. Exp. Appl. Acarol. 27:241-251.

Nolan I. 1990. Acaricide resistence in single and multi-host ticks and strategies for control. Parasitologia, Roma, 32(1):145-153.

Pereira J.P. 2006. Eficácia in vitro de formulações comerciais de carrapaticidas em teleóginas de Boophilus microplus coletadas de bovinos leiteiros do Vale do Paraíba, estado de São Paulo. Revta Bras. Parasitol. Vet. 15(2):45-48.

Rocha C.M.B.M., Oliveira P.R., Leite R.C., Cardoso L.C., Calic S.B. \& Furlong J. 2006. Percepção dos produtores de leite do município de
Passos, MG, sobre o carrapato Boophilus microplus (Acari: Ixodidae), 2001. Ciência Rural 36(4):1235-1242.

Silva M.C.L., Neves Sobrinho R. \& Linhares G.F.C. 2000. Avaliação in vitro da eficácia do Clorfenvinfós e da Cialotrina sobre o Boophilus microplus colhidos em bovinos da bacia leiteira da microrregião de Goiânia, Goiás. Ciênc. Anim. Bras., Goiânia, 1(2):143-148.

Souza A.P., Sartor A.A., Bellato V. \& Perussolo S. 2003. Eficácia de carrapaticidas em rebanhos de bovinos leiteiros de municípios da região centro sul do Paraná. Revta Ciênc. Agrovet., Lages, 2:245-250.

Sutherst R.W. \& Comins H.N. 1997. The management of acaricide resistance in the cattle tick Boophilus microplus (Canestrini) (Acari: Ixodidae) in Australia. Bull. Entomol. Res. 69:519-540.

Vargas M.S., Céspedes N.S., Sánchez H.F., Martins J.R. \& Céspedes C.O.C. 2003. Avaliação in vitro de uma cepa de campo de Boophilus microplus (Acari: Ixodidae) resistente à Amitraz. Ciência Rural 33(4):737-742.

Vivas R.I.R., Días M.A.A., Arevalo F.R., Sanchez H.F., Santamaría V.M. \& Cruz R.R. 2006. Prevalence and potencial risk factores for organophosphate and pyrethroid resistance in Boophilus microplus ticks on cattle ranches from the State of Yucatan, México. Vet. Parasitol. 136:335-342. 